Corresponding author.

Árpád Bürmen

Faculty of Electrical Engineering

Tržaška cesta 25

SI-1000 Ljubljana

Slovenia

E-mail: arpadb@fides.fe.uni-lj.si 


\title{
EXTENDED GLOBAL CONVERGENCE FRAMEWORK FOR UNCONSTRAINED OPTIMIZATION
}

\author{
Árpád Bürmen \\ Franc Bratkovič \\ Janez Puhan \\ Iztok Fajfar \\ Tadej Tuma \\ University of Ljubljana \\ Faculty of Electrical Engineering \\ Tržaška cesta 25 \\ SI-1000 Ljubljana \\ Slovenia
}

\begin{abstract}
An extension of the global convergence framework for unconstrained derivative free optimization methods is presented. The extension makes it possible for the framework to include optimization methods with varying cardinality of the ordered direction set. Grid-based search methods are shown to be a special case of the more general extended global convergence framework. Furthermore, the required properties of the sequence of ordered direction sets listed in the definition of grid-based methods are relaxed and simplified by removing the requirement of structural equivalence.
\end{abstract}

Keywords. Derivative free optimization, descent methods, convergence analysis, grid-based optimization.

1991 Mathematics Subject Classification. 49M30, 65K05.

Abbreviated title. Extended global convergence framework. 
1. Introduction. Solving unconstrained optimization problems $\min _{x \in \mathbb{R}^{n}} f(x), f: \mathbb{R}^{n} \rightarrow \mathbb{R}$ is an important issue in engineering design. Since most tools used for calculating the value of the cost function $f(x)$ provide no gradient information or this information is unreliable or incomplete, one must resort to utilizing direct search techniques. Direct search is currently going through a renaissance due to many general convergence results published for various algorithms in the last 10 years (see [1], [2], and [3] for an overview). The ideas from [4] and [5] seeded the definition and the convergence theory for the class of pattern search methods (PS) [6]. Two more general convergence theories for unconstrained optimization followed: global convergence framework for derivative free optimization methods (GCF) by Lucidi and Sciandrone [7], which emerged as an attempt to unify convergence theories for pattern search and line search (LS) [8], and grid-based search (GBS) [9] as an extension of PS.

All of the above mentioned methods are descent methods meaning that they produce a sequence of points with nonincreasing cost function value. The goal towards which all these methods strive is to find some point in the search space where the gradient of the cost function is 0 regardless of the initial point provided to the algorithm. In an iteration the cost function value is probed at a set of points lying on rays emanating from the current point. Based on the results of this search the current point and possibly some step information is updated upon which some finite process can be executed in order to find further decrease in the cost function value. The aforementioned steps are iterated until some stopping criterion is met. The vectors that define the rays must fulfill certain conditions. In case of PS and GBS the conditions are based on the notion of a positive base. In the GCF a somewhat different and more general requirement is imposed upon the vectors. It was shown in [7] that positive bases fulfill this requirement.

A major restriction regarding the set of search directions in PS is that they must be a member of a finite set, thereby limiting the capabilities of the algorithm to adapt to the fitness landscape by updating the search directions. Another limitation applies to the step length and requires it to be rational. These two restrictions guarantee that all probed points lie on a rational lattice, which is refined as the search advances. This underlying algebraic structure is the key to the convergence proof in [6].

GBS is an extension of PS. The first major improvement over PS is the milder algebraic structure imposed on the iterates, i.e. the set of available search directions can be infinite. It allows construction of 
algorithms with finite termination properties on quadratics [10] that still keep the robustness of pattern search. The second improvement is the possibility to execute some finite process at the end of an iteration that attempts to find further decrease in cost function value thus providing the possibility to incorporate other minimization strategies to accelerate the search.

This paper deals with the properties of GBS viewed from the perspective of convergence conditions set forth by Lucidi and Sciandrone. The convergence properties of GBS are shown to be a direct result of the GCF convergence theorem thus proving that GBS is just another special case from the class of methods covered by GCF.

In the following sections the extended global convergence framework based on [7] is presented. An overview of properties of positive bases is given and the feasibility of a sequence of ordered positive bases with varying cardinality is demonstrated. Grid-based search algorithms are presented and their properties are demonstrated to fulfill the requirements of the extended global convergence framework. The requirements on the sequence of ordered direction sets used by GBS are simplified by removing the requirement of structural equivalence.

Notation. Let $|\mathcal{A}|$ denote the cardinality of $\mathcal{A},\|x\|$ the euclidean norm of $x$, and $\mathcal{L}_{f}(x)=\{y$ : $f(y) \leq f(x)\}$ the level set of $f(x)$. Let $o(x)$ be a function for which $\lim _{x \rightarrow 0} \frac{o(x)}{x}=0 . \mathbb{R}, \mathbb{Z}, \mathbb{Z}^{+}$, and $\mathbb{Q}$ denote real, integer, positive integer, and rational numbers, respectively.

2. An overview of GCF. All convergence properties of algorithms conforming to GCF are valid when $f(x)$ is continuously differentiable.

Optimization algorithms conforming to GCF utilize a set of search directions in order to explore the search space and drive their decisions. The following definitions establish the notion of an ordered direction set and a feasible sequence of ordered direction sets.

DeFINITION 2.1. An ordered direction set is an ordered set of vectors

$$
\mathcal{P}=\left\{p^{i}: p^{i} \neq 0, \quad i=1, \ldots, r\right\}
$$

Definition 2.2. A feasible sequence of ordered direction sets $\left\{\mathcal{P}_{k}\right\}$, is such that 


$$
\max _{k}\left|\mathcal{P}_{k}\right|=N<\infty
$$

and for any given sequence of points $\left\{x_{k}\right\}$

$$
\lim _{k \rightarrow \infty}\left\|\nabla f\left(x_{k}\right)\right\|=0
$$

if and only if

$$
\lim _{k \rightarrow \infty} \sum_{i=1}^{\left|\mathcal{P}_{k}\right|} \min \left(0, \frac{\nabla f\left(x_{k}\right)^{T} p_{k}^{i}}{\left\|p_{k}^{i}\right\|}\right)=0
$$

where $p_{k}^{i}$ denotes the $i$-th vector from the $k$-th element of the sequence $\left\{\mathcal{P}_{k}\right\}$.

Note that all $\mathcal{P}_{k}$ have the same cardinality according to [7]. Sets of search directions utilized by positive basis pattern search and rank ordered pattern search [11] are feasible. Further, any bounded sequence of direction sets of the same cardinality with every cluster point positively spanning $\mathbb{R}^{n}$, is feasible. See [7] for the proof.

The set of global convergence conditions for derivative free unconstrained optimization algorithms is given by the following theorem which is an extension of the convergence theorem from [7] in the sense that it doesn't require the same cardinality for all ordered direction sets:

THEOREM 2.3. Suppose that there exists a compact set $\mathcal{C}_{0}$ such that the level set $\mathcal{L}_{f}\left(x_{0}\right) \subseteq \mathcal{C}_{0}$. Let $\mathcal{A}$ denote a derivative free unconstrained optimization algorithm, $\mathcal{A}\left(x_{0}\right)=\left\{x_{k}\right\}$ the sequence of points produced by $\mathcal{A}$ starting from the initial point $x_{0}$ and $\left\{\mathcal{P}_{k}\right\}$ a feasible sequence of ordered direction sets. If the following conditions hold:

1. the sequence of function values at points produced by $\mathcal{A}$ is nonincreasing.

$$
f\left(x_{k+1}\right) \leq f\left(x_{k}\right),
$$

2. for every pair $(k, i), k \in \mathbb{Z}^{+}, i \in\left\{1, \ldots,\left|\mathcal{P}_{k}\right|\right\}$, there exists an index $h_{k}^{i}$ and a scalar $\xi_{k}^{i}>0$, such that

$$
\begin{gathered}
f\left(x_{h_{k}^{i}}+\xi_{k}^{i} p_{k}^{i}\right) \geq f\left(x_{h_{k}^{i}}\right)+o\left(\xi_{k}^{i}\left\|p_{k}^{i}\right\|\right) \\
\lim _{k \rightarrow \infty} \sum_{i=1}^{\left|\mathcal{P}_{k}\right|} \xi_{k}^{i}\left\|p_{k}^{i}\right\|=0
\end{gathered}
$$




$$
\lim _{k \rightarrow \infty} \sum_{i=1}^{\left|\mathcal{P}_{k}\right|}\left\|x_{k}-x_{h_{k}^{i}}\right\|=0
$$

Then,

$$
\lim _{k \rightarrow \infty}\left\|\nabla f\left(x_{k}\right)\right\|=0
$$

Proof. We proceed by a different argument than Lucidi and Sciandrone [7]. Our argument is inspired by $[9]$.

From (2) it follows for all $i=1, \ldots,\left|\mathcal{P}_{k}\right|$ :

$$
\begin{aligned}
f\left(x_{h_{k}^{i}}+\xi_{k}^{i} p_{k}^{i}\right)-f\left(x_{h_{k}^{i}}\right) & =\int_{0}^{\xi_{k}^{i}}\left(\nabla f\left(x_{h_{k}^{i}}+t p_{k}^{i}\right)-\nabla f\left(x_{h_{k}^{i}}\right)+\nabla f\left(x_{h_{k}^{i}}\right)\right)^{T} p_{k}^{i} d t= \\
& =\xi_{k}^{i} \nabla f\left(x_{h_{k}^{i}}\right)^{T} p_{k}^{i}+E_{k}^{i} \geq o\left(\xi_{k}^{i}\left\|p_{k}^{i}\right\|\right) \\
E_{k}^{i} & =\int_{0}^{\xi_{k}^{i}}\left(\nabla f\left(x_{h_{k}^{i}}+t p_{k}^{i}\right)-\nabla f\left(x_{h_{k}^{i}}\right)\right)^{T} p_{k}^{i} d t
\end{aligned}
$$

Due to (1) all iterates $x_{k}$ lie in $\mathcal{L}_{f}\left(x_{0}\right) \subseteq \mathcal{C}_{0}$. Since $\mathcal{C}_{0}$ is compact and $f(x)$ is continuously differentiable, $\nabla f(x)$ is Lipschitz continuous on $\mathcal{L}_{f}\left(x_{0}\right)$ with Lipschitz constant $C$.

$$
\|\nabla f(y)-\nabla f(x)\| \leq C\|y-x\|
$$

Now we can see that $E_{k}^{i}$ is bounded.

$$
\left|E_{k}^{i}\right| \leq \int_{0}^{\xi_{k}^{i}} C t\left\|p_{k}^{i}\right\|^{2} d t=\frac{1}{2} C\left(\xi_{k}^{i}\left\|p_{k}^{i}\right\|\right)^{2} \quad i=1, \ldots,\left|\mathcal{P}_{k}\right|
$$

By joining (6) and (7) we get

$$
\xi_{k}^{i} \nabla f\left(x_{h_{k}^{i}}\right)^{T} p_{k}^{i}+\frac{1}{2} C\left(\xi_{k}^{i}\left\|p_{k}^{i}\right\|\right)^{2} \geq o\left(\xi_{k}^{i}\left\|p_{k}^{i}\right\|\right) \quad i=1, \ldots,\left|\mathcal{P}_{k}\right|
$$

Due to (1) the sequence $\left\{x_{k}\right\}$ belongs to the compact level set $\mathcal{C}_{0} \supseteq \mathcal{L}_{f}\left(x_{0}\right)$ and thus admits at least one cluster point. Let $x_{\infty}$ be any cluster point of $\left\{x_{k}\right\}$. There exists an infinite set of indices $K_{1} \subseteq \mathbb{Z}^{+}$ such that:

$$
\lim _{k \rightarrow \infty, k \in K} x_{k}=x_{\infty}
$$

Since the cardinality of any ordered direction set from the sequence $\left\{\mathcal{P}_{k}\right\}$ is bound from above, there exist an infinite subsequence of indices $K_{2} \subseteq K_{1}$ such that $\left|\mathcal{P}_{k}\right|=N_{1} \leq N$ for all $k \in K_{2}$. Since all 
$p_{k}^{i} /\left\|p_{k}^{i}\right\|$ belong to a compact set ( $n$ dimensional sphere), there exists an infinite subsequence of indices $K \subseteq K_{2}$ such that

$$
\lim _{k \rightarrow \infty, k \in K} \frac{p_{k}^{i}}{\left\|p_{k}^{i}\right\|}=p_{\infty}^{i} \quad i=1, \ldots, N_{1}
$$

By looking at (3), (4), and regarding the fact that $\xi_{k}^{i}>0$, it is obvious that

$$
\begin{array}{r}
\lim _{k \rightarrow \infty} \max _{i=1, \ldots,\left|\mathcal{P}_{k}\right|} \xi_{k}^{i}\left\|p_{k}^{i}\right\|=0, \\
\lim _{k \rightarrow \infty} \max _{i=1, \ldots,\left|\mathcal{P}_{k}\right|}\left\|x_{k}-x_{h_{k}^{i}}\right\|=0 .
\end{array}
$$

Now take the limit of (8) as $k \rightarrow \infty, k \in K$. Considering the definition of $o(x)$, and (11) we get

$$
\lim _{k \rightarrow \infty, k \in K} \nabla f\left(x_{h_{k}^{i}}\right)^{T} \frac{p_{k}^{i}}{\left\|p_{k}^{i}\right\|} \geq 0 \quad i=1, \ldots, N_{1} .
$$

By considering (10), (12), and continuity of $\nabla f(x)$

$$
\nabla f\left(x_{\infty}\right)^{T} p_{\infty}^{i} \geq 0 \quad i=1, \ldots, N_{1}
$$

Obviously also

$$
\sum_{i=1}^{N_{1}} \min \left(0, \nabla f\left(x_{\infty}\right)^{T} p_{\infty}^{i}\right)=0 .
$$

According to Definition 2.2 we have

$$
\left\|\nabla f\left(x_{\infty}\right)\right\|=0
$$

Since $x_{\infty}$ is any cluster point of $\left\{x_{k}\right\}$

$$
\lim _{k \rightarrow \infty}\left\|\nabla f\left(x_{k}\right)\right\|=0
$$

$\square$

Note that Theorem 2.3 guarantees that the gradient at the calculated iterates approaches 0 as the number of iterations approaches infinity. However there is no guarantee that the sequence of iterates $\left\{x_{k}\right\}$ will converge. 
3. Positive bases and GCF. Some basic information regarding positive bases and their relation to GCF is given. An interested reader can find details in [12]. Positive bases play a key role in defining the sets of points examined by grid-based search algorithms.

Definition 3.1. The set of vectors $\left\{a_{1}, \ldots, a_{r}\right\}$ is positively independent if none of its members can be written as a nonnegative linear combination of the others.

Definition 3.2. The positive span of a set of vectors $\mathcal{P}=\left\{a_{1}, \ldots, a_{r}\right\}$ is a set of vectors $\{a: a=$ $\left.c_{1} a_{1}+\ldots+c_{r} a_{r}, \quad c_{1}, \ldots, c_{r} \geq 0\right\} . \mathcal{P}$ is a positive base for $\mathbb{R}^{n}$ if it is positively independent and its positive span equals $\mathbb{R}^{n}$.

LEMMA 3.3. If $\mathcal{P}$ is a positive base for $\mathbb{R}^{n}$ then $n+1 \leq|\mathcal{P}| \leq 2 n$.

The interested reader can refer to [12] for a proof.

Lemma 3.4. Any sequence of ordered positive bases $\left\{\mathcal{P}_{k}\right\}$ where $\left|\mathcal{P}_{k}\right|=r \geq n+1$ where all directions are bounded $\left(\left\|p_{k}^{i}\right\| \leq M, i=1, \ldots, r\right)$ and all cluster points of the sequence positively span $\mathbb{R}^{n}$ is feasible.

The proof can be found in [7].

Lemma 3.5. Any sequence of ordered direction sets $\left\{\mathcal{P}_{k}\right\}, n+1 \leq\left|\mathcal{P}_{k}\right| \leq N$, with every direction satisfying $\left\|p_{k}^{i}\right\| \leq M$ and all cluster points being a positive base for $\mathbb{R}^{n}$ is feasible.

Proof. We begin by splitting the sequence $\left\{\mathcal{P}_{k}\right\}$ in subsequences containing ordered direction sets of the same cardinality

$$
\left\{\mathcal{P}_{k}\right\}=\left\{\mathcal{P}_{k}\right\}_{k \in K_{1}} \cup \ldots \cup\left\{\mathcal{P}_{k}\right\}_{k \in K_{j}}
$$

Since $\left\{\mathcal{P}_{k}\right\}$ is infinite at least one of the subsequences obtained by splitting $\left\{\mathcal{P}_{k}\right\}$ is infinite. Let $\left\{\mathcal{P}_{k}\right\}_{k \in K_{l}}$ be any such infinite subsequence. Due to boundness of its members it admits at least one cluster point. Since all cluster points of $\left\{\mathcal{P}_{k}\right\}$ and therewith $\left\{\mathcal{P}_{k}\right\}_{k \in K_{l}}$ positively span $\mathbb{R}^{n}$ the subsequence is feasible (Lemma 3.4). By taking into account the fact that $\left\{\mathcal{P}_{k}\right\}_{k \in K_{l}}$ is any such infinite subsequence, the sequence $\left\{\mathcal{P}_{k}\right\}$ is feasible.

Definition 3.6. Two ordered direction sets $\mathcal{V}=\left\{v_{j}\right\}$ and $\mathcal{W}=\left\{w_{j}\right\}$ are structurally equivalent in $\mathbb{R}^{n}$ if and only if $|\mathcal{V}|=|\mathcal{W}|$, and

$$
i=n+1, \ldots,|\mathcal{V}| \quad v_{i}=\sum_{j=1}^{n} \beta_{i j} v_{j} \Leftrightarrow w_{i}=\sum_{j=1}^{n} \beta_{i j} w_{j}
$$


The notion of structural equivalence is used along with several other requirements by GBS to guarantee that the positive bases in the sequence don't become arbitrarily "bad" (i.e. all cluster points of the sequence are positive bases for $\mathbb{R}^{n}$ ).

4. Grid-based search. The basic idea of grid-based search is to look for grid local minimizers on consecutive grids.

Definition 4.1. A grid is a set of points defined by origin $y$, rational positive base $\mathcal{P}=\left\{p^{i}, i=\right.$ $1, \ldots, r\}$, and step length $h$

$$
\mathcal{G}(y, \mathcal{P}, h)=\left\{x: x=y+h \sum_{i=1}^{r} \alpha_{i} p^{i}, \quad \alpha_{1}, \ldots, \alpha_{r} \in \mathbb{Z}\right\}
$$

The notion of a grid makes sense only if all $p \in \mathcal{P}$ are rational $\left(p \in \mathbb{Q}^{n}\right)$. Rationality ensures that the intersection between any grid and any bound subset of $\mathbb{R}^{n}$ is finite. It also represents the key element in the finiteness proof of the inner loop for GBS and PS. Grid-based search algorithms use positive bases to explore the search space. The main idea is to produce a sequence of grid local minimizers with increasing grid resolution.

Definition 4.2. A grid local minimizer $\check{x} \in \mathcal{G}(y, \mathcal{P}, h)$ is a point where

$$
f\left(\breve{x}+h p^{i}\right) \geq f(\check{x}) \quad i=1, \ldots,|\mathcal{P}|
$$

The core of the algorithm is the finite search for a grid local minimizer in $n$ dimensional space (step 2). The proof of finiteness for step 2 can be found in [9] and follows the same argument as in [5] and [6]. After a grid local minimizer is found an arbitrary finite search is conducted (step 3) in order to improve the function value found in step 2. The better one of the two results (grid local minimizer, outcome of the finite search process) becomes the new iterate and also the origin of a new grid defined by a possibly new positive base and updated step length parameter (steps 4, 5, and 1). 


\section{GRID-BASED SEARCH}

Set grid counter $k=1$ and iteration counter $j=1$.

Set initial grid origin $y_{1}$ and initial point $x_{1}$ to $x_{0}$.

while (stopping criterion false) do

1. Chose step a length parameter $h_{k}$ and a positive base $\mathcal{P}_{k}=\left\{p_{k}^{l}\right.$ : $\left.l=1, \ldots, c_{k}\right\}$. Set $i=1$ and $q=0$.

2. while $\left(q<\left|\mathcal{P}_{k}\right|\right)$ do

a) Evaluate $f(x)$ at a finite number of points on the grid $\mathcal{G}\left(y_{k}, \mathcal{P}_{k}, h_{k}\right)$, including but not limited to $x_{j}+h_{k} p_{k}^{i}$.

b) If some point $x^{*}$ with $f\left(x^{*}\right)<f\left(x_{j}\right)$ is found, set $x_{j+1}=x^{*}$, $j=j+1$, and $q=0$. Otherwise increment $q$.

c) $i=i+1$.

d) If $i>\left|\mathcal{P}_{k}\right|$, set $i=1$.

end

3. Execute a finite process resulting in point $z_{k}$.

4. Let $x_{j+1}=y_{k+1}= \begin{cases}x_{j} & f\left(z_{k}\right) \geq f\left(x_{j}\right) \\ z_{k} & f\left(z_{k}\right)<f\left(x_{j}\right)\end{cases}$

5. $j=j+1, k=k+1$.

end

5. Grid-based search in GCF. We start by listing the conditions imposed on the grid-based search algorithm. Then we demonstrate that the sequence of positive bases in GBS is feasible. Finally the relationship between GBS and GCF is shown.

According to [9] the function must be continuously differentiable and the sequence of iterates bounded. The step length control is not specified in the algorithm. The only requirement imposed upon the step length parameter $h$ is that $\lim _{k \rightarrow \infty} h_{k}=0$.

The following lemma is about the feasibility of the sequence of ordered direction sets used in GBS. The requirements imposed upon the directions are the same as in [9].

Lemma 5.1. If the sequence of ordered direction sets $\left\{\mathcal{P}_{k}\right\}$ used by GBS to search through $\mathbb{R}^{n}$ satisfies 
the following conditions

1. there exist a positive constant $M$ such that $\left\|p_{k}^{i}\right\| \leq M$ for all $k, i=1, \ldots,\left|\mathcal{P}_{k}\right|$,

2. there exist a positive constant $\kappa$ such that $\left|\operatorname{det}\left(p_{k}^{1}, \ldots, p_{k}^{n}\right)\right| \geq \kappa$ for all $k$,

3. there exists a finite subset of positive bases $\mathcal{S}$ from $\left\{\mathcal{P}_{k}\right\}$ such that all members of $\left\{\mathcal{P}_{k}\right\}$ are structurally equivalent to some member of $\mathcal{S}$,

then it is feasible.

Proof. Split the sequence of ordered direction sets into subsequences of ordered direction sets with constant cardinality (same approach as in Lemma 3.5). Now take any such subsequence $\left\{\mathcal{P}_{k}\right\}_{k \in K_{l}}$. Due to the first requirement it is bounded and therefore admits at least one cluster point. Due to the second requirement, the first $n$ directions are linearly independent for all members of the subsequence $\left\{\mathcal{P}_{k}\right\}_{k \in K_{l}}$ and also for any of its cluster points. The third requirement assures us that all members and all cluster points are structurally equivalent to some positive base from $\mathcal{S}$. The second and the third requirement therefore guarantee that all cluster points of $\left\{\mathcal{P}_{k}\right\}_{k \in K_{l}}$ are positive bases for $\mathbb{R}^{n}$. Since this is valid for any of the subsequences obtained by splitting $\left\{\mathcal{P}_{k}\right\}$, Lemma 3.5 assures us that the ordered direction set sequence $\left\{\mathcal{P}_{k}\right\}$ is feasible.

By looking back at Lemma 3.5 we can see that the requirements of Lemma 5.1 can be relaxed. We only need to keep the boundness of the directions (requirement 1) and all cluster points of the sequence must positively span $\mathbb{R}^{n}$. There is no need to require any kind of structural equivalence. Although it is a mild requirement it still limits the capability of the search directions to adapt to the local behavior of the cost function.

THEOREM 5.2. The sequence of grid local minimizers produced by a grid-based search algorithm $\left\{y_{k}\right\}$ is such that $\lim _{k \rightarrow \infty}\left\|\nabla f\left(y_{k}\right)\right\|=0$.

Proof. Since the GBS requires the boundness of the sequence of iterates $\left\{x_{j}\right\}$, there exists a compact set $\mathcal{C}_{0}$ containing the whole sequence of iterates. The sequence of iterates and therewith the sequence of grid local minimizers is nonincreasing due to steps $2 \mathrm{~b}$ and 4 of the algorithm, so (1) from Theorem 2.3 holds and the level set $\mathcal{L}_{f}\left(x_{0}\right)$ lies in the compact set $\mathcal{C}_{0}$.

Choose $h_{k}^{i}=k$. Then the positive base $\mathcal{P}_{k}$ used to construct a grid with its origin at $y_{k}$ is actually 
the direction set that applies to $y_{k+1}$ from the sequence of grid local minimizers $\left\{y_{k}\right\}$ when the sequence is viewed through Theorem 2.3. This positive base is used to find a new grid local minimizer starting at point $y_{k}$. When a grid local minimizer is found $\left(y_{k+1}\right)$, we can say (by looking back at Definition 4.2 )

$$
f\left(y_{k+1}+h_{k} p_{k}^{i}\right) \geq f\left(y_{k+1}\right), \quad i=1, \ldots,\left|\mathcal{P}_{k}\right|
$$

Now say that $o(x)=0$ and $\xi_{k}^{i}=h_{k}, i=1, \ldots,\left|\mathcal{P}_{k}\right|$. It becomes obvious that (13) is an equivalent of (2) from Theorem 2.3.

By recalling the fact that all directions appearing throughout the grid-based search as members of ordered positive bases are bounded $\left(\left\|p_{k}^{i}\right\| \leq M\right.$, see proof of Lemma 5.1) and the fact that grid-based search requires $\lim _{k \rightarrow \infty} h_{k}=0,(3)$ of Theorem 2.3 also holds.

Finally since we chose $h_{k}^{i}=k,\left\|y_{k}-y_{h_{k}^{i}}\right\|=0$ and (4) of Theorem 2.3 holds.

Since all requirements of Theorem 2.3 are fulfilled and the sequence of ordered positive bases used in GBS is feasible,

$$
\lim _{k \rightarrow \infty}\left\|\nabla f\left(y_{k}\right)\right\|=0
$$

6. Conclusions. The extended global convergence framework is a powerful tool for analyzing the convergence properties of existing and developing new globally convergent derivative-free optimization algorithms. The introduced extension allows the analysis of algorithms with varying cardinality of the ordered search direction set. The extended global convergence framework could provide means for proving the convergence of parallel algorithms where the number of search directions changes due to failures caused by errors in the communication between the search processes or due to failures of the search processes.

The grid based search has been proved to conform to the extended global convergence framework. Although the structural equivalence of all ordered search direction sets to some finite number of positive bases is required by the original grid-based search algorithm, the extended framework allows any sequence of positive bases with all cluster points being positive bases themselves, to be used. This removes the structural equivalence requirement and enhances the flexibility of the family of grid-based search. 
Acknowledgments. The authors wish to thank M. Sciandrone for the electronic version of the technical report on which [7] is based.

\section{REFERENCES}

[1] Lewis, R. M., Torczon, V., And Trosset, M. W., Direct search methods: then and now, Journal of Computational and Applied Mathematics, 1-2 (2000), 191-207.

[2] Wright, M. H., Direct search methods: once scorned, now respectable, in Griffiths, D. F., Watson, G. A., Numerical Analysis 1995, Addison-Wesley Longman, Reading, MA, (1996), 191-208.

[3] Powell, M. J. D., Direct search algorithms for optimization calculations, Acta Numerica, (1998), 287-336.

[4] Yu, Wen-CI, Positive basis and a class of direct search techniques, Scientia Sinica, Special Issue of Mathematics, 1 (1979), 53-67.

[5] Torczon, V., Multi-directional search: A direct search method for parallel machines, Ph.D. thesis, Department of Mathematical Sciences, Rice University, Houston, TX, 1989.

[6] Torczon, V., On the convergence of pattern search algorithms, SIAM Journal on Optimization, 7 (1997), 1-25.

[7] Lucidi, S. And Sciandrone, M., On the Global Convergence of Derivative-Free Methods for Unconstrained Optimization, SIAM Journal on Optimization, 13 (2002), 97-116.

[8] Grippo, L., Lampariello, F., And Lucidi, S., Global convergence and stabilization of unconstrained minimization methods without derivatives, Journal of Optimization Theory and Applications, 3 (1988), 385-406.

[9] Coope, I. D. And Price, C. J., On the convergence of grid-based methods for unconstrained optimization, SIAM Journal on Optimization, 4 (2001), 859-869.

[10] Coope, I. D. And Price, C. J., A direct search conjugate directions algorithm for unconstrained minimization, Tech. Rep. 188, Department of Mathematics \& Statistics, University of Canterbury, Christchurch, New Zealand, 1999.

[11] Lewis, R. M. And Torczon, V., Rank ordering and positive bases in pattern search algorithms, Tech. Rep. 9671, Institute for Computer Applications in Science and Engineering (ICASE), NASA Langley Research Center, Hampton, VA, (1996).

[12] Davis, C., Theory of positive linear dependence, American Journal of Mathematics, (1954), 83-108. 